\title{
Settling a claim
}

Jane Merivale

Senior Dento-legal advisor, BDA Indemnity

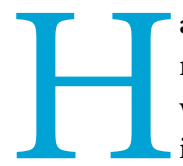
ave you ever found yourself at rush hour, standing in the rain waiting for a bus and wondering if you will manage to get to the station in time to catch your train? Then out of the blue a taxi comes along by with its 'For Hire' light on. Suddenly your choices for achieving the desired onward journey are improved. It might cost more to take a taxi, but the time saved and the certainty provided by a driver with 'the knowledge' may well be worth it - particularly if the journey is important.

Patient journeys are very similar. It is important that the patient experience is a good one, and that they are satisfied with their treatment. In today's culture there is an unfortunate attitude that when something isn't satisfactory or indeed the outcome isn't what was anticipated or hoped for, then someone must be at fault and 'someone' or some organisation will have to pay, either literally with financial redress or reputationally.

The dental team should not hesitate to seek professional advice if the patient's complaint cannot be resolved. Indeed a poorly worded response is difficult to undo and can be professionally embarrassing if aired online by the patient. That's why your choice of indemnity provider is so important. Do you want the faster taxi ride that may involve refunding fees, or would you prefer to stand your ground, take a longer and sometimes less predictable bus ride and maybe miss the train just to demonstrate that you did the

right thing?

If you have BDA Indemnity, the BDA's

dentolegal advisors will initially assess the complaint against the patient's records.

The skill is to 'horizon spot': to assess if the complaint has merit and where it may land if it is not resolved at the earliest opportunity. This assessment relies on both clinical expertise and the experience of having handled many complaints. From the available information the adviser will looks at what has happened (the treatment or lack of) and how the care has been delivered. A similar process is followed by most indemnity providers.

The technical focus is to identify any element of the clinical treatment that could be regarded as below a reasonable standard (a breach in the duty of care) from which some problem has resulted (causation). If so, then the advice would be that the complaint has the makings of a claim if the patient decided to instruct a solicitor. The records would then be subject to scrutiny by an independent expert.

The complaint is also evaluated to see if there is any aspect that could give rise to criticism should the patient draw their concerns to the attention of the regulator. The focus then is more on how the treatment was provided; the attitude and behaviour of the registrant, as well as the treatment itself.

Early resolution in either scenario is advised in an effort to resolve the complaint at a local level, and to hopefully avert escalation along either of the above routes. However, nothing is ever quite that straightforward and there are times when the complainant is dissatisfied and none of the above vulnerability is identified. What then? between escalation and resolution. Even when a defence might be justified, when the defence process is explained and the time and stress added in, the clinician may well prefer to take a taxi rather than the bus and offer a refund. Colleagues often change their mind.

We also hear those who say, 'I don't think I have done anything wrong, so I will certainly not give in and make a refund. Here the concept of the 'no negligence loser' is significant. In a sense, someone has to defuse rather than aggravate the situation, and make every effort to draw the unhappy situation to a close. A failure to do so could cost time whilst a third party explores the issues raised, additional money and a loss of practice goodwill and local reputation. Human nature being what it is, the facts are likely to be shared inaccurately and exaggerated.

And so to the concept of symbolic atonement: giving the patient something to make up for the problem they've experienced. That something would usually be a refund of fees as a goodwill gesture, with no admission of fault, and accompanied by a kindly worded explanation. Research shows that a complaint handled well often enhances reputation and leads to customer loyalty.

There are a variety of reasons why a refund of fees might be advised, and the suggestion will always be given with members' best interests in mind.

https://doi.org/10.1038/s41404-020-0328-5

\section{Refund demand}

If it is thought that the complaint can be successfully defended, the clinician will be offered every assistance in achieving that outcome.

A well drafted letter, explaining the care provided (supported by the contemporaneous records) and an apology that the person has found the need to complain may suffice, or it may not; the complaint remains and the demand for a refund is all that sits

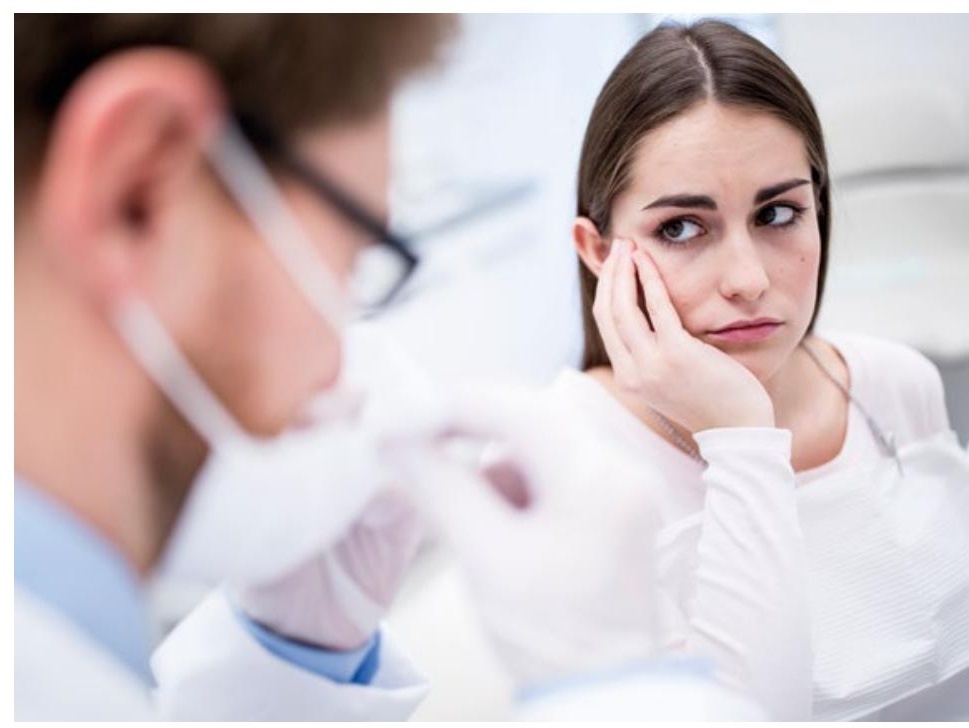

